\title{
ANÁLISE DO PERFILEPIDEMIOLÓGICO DAS INTERNAÇÕES EM UMA UNIDADE DE TERAPIA INTENSIVA MATERNA
}

Sandra Taveiros de Araújo1, Maria Elisângela Torres de Lima Sanches ${ }^{1}$ Wanubia Santos Nascimento²

Objetivo: analisar o perfil epidemiológico das pacientes internadas em uma Unidade de Terapia Intensiva (UTI) materna. Metodologia: Estudo transversal retrospectivo quantitativo, com 70 pacientes internas em uma UTI materna do Estado de Alagoas, Maternidade Santa Mônica (MESM), no ano 2015. Resultados: Os três principais diagnósticos das internações foram as sindromes hipertensivas, sindromes hemorrágicas e cardiopatia. Média de idade 27 anos, a maioria procedente do interior de Alagoas. 48,5\% primigesta, taxa de cesárea 94,7\%. Procedimentos cirúrgicos mais frequentes foram laparotomia, intubação orotraqueal, e histerectomia. Duração do internamento de 1-31 dias. Taxa de óbito 5,7\%. Conclusão: o perfil epidemiológico das pacientes do estudo diverge, em alguns aspectos, de outras pesquisas, sendo elevada, principalmente, as taxas de cesárea e óbito materno, é contraditório também as principais causas de internamento. Assim, a assistência obstétrica no estado de Alagoas precisa avançar para diminuir os óbitos maternos e reduzir a taxa de cesárea.

Descritores: Gravidez de alto risco, Unidade de Terapia Intensiva, Mortalidade Materna.

\section{ANALYSIS OF THE EPIDEMIOLOGICAL PROFILE OF HOSPITALIZATIONS IN A MATERNAL INTENSIVE CARE UNIT}

Objective: to analyze the epidemiological profile of patients admitted to a Maternal Intensive Care Unit (ICU). Methodolofy: A retrospective quantitative cross-sectional study with 70 inpatients at a maternal ICU in the State of Alagoas, Santa Mônica Maternity (MESM), in the year 2015. Results: The three main diagnoses of hospitalizations were hypertensive syndromes, hemorrhagic syndromes and cardiopathy. Average age 27 years, most coming from the interior of Alagoas. $48.5 \%$ primigravida, caesarean section $94.7 \%$. Most frequent surgical procedures were laparotomy, orotracheal intubation, and hysterectomy. Length of stay from 1-31 days. Death rate 5.7\%. Conclusion: The epidemiological profile of the patients in the study diverges, in some aspects, from other studies, mainly due to the rates of maternal caesarean section and death, which is also contradictory to the main causes of hospitalization. Thus, obstetric care in the state of Alagoas needs to move forward to reduce maternal deaths and reduce the rate of cesarean section.

Descriptors: High risk pregnancy, Intensive care unit, Maternal Mortality

\section{ELANÁLISIS DEL PERFILEPIDEMIOLÓGICO DE LAS HOSPITALIZACIONES EN UNA UNIDAD DE CUIDADOS INTENSIVOS MATERNAL} Objetivo: Analizar el perfil epidemiológico de los pacientes hospitalizados en una madre Unidad de Cuidados Intensivos (UCI). Metodología: Estudio retrospectivo transversal cuantitativo con 70 pacientes en una UCI materna interna del Estado de Alagoas, maternidad Santa Mónica (HSHAV) en el año 2015. Resultados: Los tres diagnósticos principales de admisión eran hipertensos trastornos, hemorragia y de la enfermedad cardiaca sindromes. Media de edad 27 años, la mayoría procedente del interior de Alagoas. 48,5\% primigesta, tasa de cesárea 94,7\%. Los procedimientos quirúrgicos más frecuentes fueron laparotomía, intubación orotraqueal, e histerectomía. Duración de la internación de 1-31 dias. Tasa de defunción 5,7\%. Conclusión: El perfil epidemiológico de los pacientes en el estudio difieren en algunos aspectos de otras investigaciones que se elevó las tasas de cesárea y sobre todo la muerte materna, es contradictorio también a las principales causas de hospitalización. Así, la asistencia obstétrica en el estado de Alagoas necesita avanzar para disminuir las muertes maternas y reducir la tasa de cesárea.

Descriptores: Embarazo de alto riesgo, Unidad de terapia intensive, La mortalidad materna. 


\section{INTRODUÇÃO}

Ainterface clínico-obstétrica assume especialimportância em se tratando de uma unidade de cuidados intensivos para pacientes no ciclo grávido-puerperal. Alterações fisiológicas próprias da gestação distinguem essas pacientes de outros adultos jovens. Diversas condições clínicas têm seu curso afetado pela gravidez e têm a interpretação de testes diagnósticos e valores laboratoriais variáveis. Além disso, complicações exclusivas da gravidez podem não ser familiares aos clínicos. Daí a importância da Unidade de Terapia Intensiva (UTI) exclusiva ao atendimento da mulher no ciclo gravídicopuerperal'.

A chance de uma mulher, durante o ciclo grávidopuerperal, ser admitida em uma Unidade de Terapia Intensiva (UTI) é bem maior do que a de uma mulher jovem, não-grávida. Estima-se que 0,1 \% a 0,9\% das gestantes desenvolvem complicações, requerendo o internamento em Unidade de Terapia Intensiva, sendo as principais indicações hipertensão, hemorragia, insuficiência respiratória e sepse ${ }^{2}$.

A maioria das mulheres admitidas em UTI tem como causa de internamento um diagnóstico obstétrico de $50 \%$ a $80 \%$. Porém, tendem a apresentar um melhor prognóstico quando comparadas às mulheres admitidas em UTI por causas clínicas. Sendo que as principais causas de morte materna são as complicações inerentes ao período gravídicopuerperal, representadas basicamente pelas complicações hipertensivas, hemorrágicas e as infecções puerperais ${ }^{3}$.

Segundo a Organização Mundial da Saúde (OMS) a definição de morte materna é a que ocorre durante a gestação ou dentro de um período de 42 dias após o término desta, independente da duração ou da localização da gravidez, devido a qualquer causa relacionada com a gravidez ou por medidas em relação a ela, porém não devidas a causas acidentais ou incidentais ${ }^{4}$. As complicações obstétricas são responsáveis por quase $50 \%$ de todas as mortes maternas no mundo, e o risco de morrer é até 25 vezes maior em países subdesenvolvidos, como o Brasil, quando comparados aos países desenvolvidos ${ }^{1}$.

Na Cúpula do Milênio da Organização das Nações Unidas (ONU), foram adotadas oito metas de desenvolvimento, nomeados Objetivos de Desenvolvimento do Milênio (ODM's), entre eles, um que era reduzir a mortalidade materna em 75\% até o ano de $2015^{5}$. Embora o progresso seja notável, a taxa de declínio anual ainda está aquém da ideal, uma vez que atingiu menos que a metade dos $75 \%$ de redução da mortalidade almejada para o período de 1990 a 2015. Apesar de não ter atingido a Meta do Milênio, a assistência obstétrica brasileira tem conseguido alguns avanços, evidenciada por índices relacionados ao pré-natal - 99\% de gestantes que passam por pelo menos uma consulta pré-natal e $73 \%$ que realizam seis ou mais consultas, conforme preconizado como ideal pelo Ministério da Saúdé.

Um acompanhamento pré-natal efetivo tem um grande impacto na redução da morbimortalidade materna desde que as mulheres tenham acesso aos serviços, os quais devem ter qualidade suficiente para identificar precocemente os fatores de riscos para a mulher e controlá-los oportunamente. Melhorar a qualidade da atenção pré-natal é essencial para prevenir esses agravos, como as mortes maternas por doenças hipertensivas, hemorragias, sepse, complicações de cesarianas não indicadas e aborto ${ }^{6}$.

Sabe-se que um bom sistema de saúde para redução da mortalidade materna deve incluir cobertura efetiva, com ampla disponibilidade, custo acessivel a todos os setores da população, além de apresentar qualidade e segurança para garantir boa assistência à saúde materna. Esses são alvos primordiais a serem atingidos por todos os sistemas de saúde, considerando-se o problema de infecções relacionadas ao parto ${ }^{7}$.

Quando essas complicações não conseguem ser evitadas, e a mulher precisa de atendimento de alta complexidade a UTI é um ambiente diferenciado, onde a paciente obstétrica requer cuidados especiais e conhecimentos específicos por parte dos profissionais, com intuito de fornecer informações para subsidiar estratégias e ações na assistência à saúde materna e proporcionar que tanto a equipe médica como a de enfermagem possam atender as necessidades das pacientes em suas especialidades. Em UTI as necessidades de cuidado de enfermagem aumentam, pois, os clientes irão precisar de avaliações críticas e rápidas, planos de cuidados abrangentes, serviços bem coordenados com outros profissionais da saúde, além de um efetivo e conveniente planejamento de alta ${ }^{8}$.

O presente estudo objetivou analisar o perfil epidemiológico das pacientes internadas na UTI materna na Maternidade Santa Mônica no ano de 2015, a fim de evidenciar os fatores de risco de maior prevalência que justificam os internamentos na UTI materna, e partir das variáveis do estudo definir o perfil clínico e o prognóstico das pacientes.

\section{METODOLOGIA}

Trata-se de um estudo transversal descritivo retrospectivo de caráter quantitativo, realizada na instituição pública, referência para alto risco, Maternidade Escola Santa Mônica (MESM), localizadas no município de Maceió-AL. Tendo como amostra censitária 70 pacientes, internas na Unidade de Terapia Intensiva (UTI) materna da MESM no ano de 2015. Os critérios de inclusão foram: mulheres internas na UTI Materna da Maternidade Escola Santa Mônica no ano de 2015. Os critérios de exclusão foram: pacientes cujo número do prontuário não foi identificado, prontuários com dados 
incompletos, ou que não estavam no Setor de Arquivo Médico e Estatística (SAME).

A coleta de dados foi realizada no período de agosto a outubro de 2016 com prontuários de pacientes internas na UTI da Maternidade Escola Santa Mônica de janeiro a dezembro de 2015 , por meio de um roteiro semiestruturado. Os princípios éticos foram seguidos na íntegra, com base na Resolução no 466/12 do Conselho Nacional de Saúde, que reporta sobre a pesquisa envolvendo seres humanos. O texto é inédito, resultado de um TCC de especialização na modalidade residência. A pesquisa teve a aprovação do Comitê de Ética e Pesquisa cujo número 1.663.601.

\section{RESULTADOS}

Após a coleta de dados, foi possivel analisar as características das pacientes internas na Unidade de Terapia Intensiva da Maternidade Escola Santa Mônica no ano de 2015 (Tabela 1). A mesma mostra que a média de idade foi de 27 anos, sendo que a maior parte das pacientes (60\%) se encontrava na faixa etária entre 20 e 34 anos de idade. A maioria tinha baixo grau de escolaridade, apenas 5,7\% com ensino superior completo, $28,5 \%$ ensino médio completo, $54,2 \%$ ensino fundamental completo, e $11,4 \%$ ignorado ou nenhuma escolaridade. Cerca de $57 \%$ era procedente de cidades do interior de Alagoas, e aproximadamente $43 \%$ de Maceió, capital do estado. Em relação à paridade, 48,5\% era primigesta, 17,1\% secundigesta, e 34,2\% multigesta.

Tabela 1. Características das pacientes internas na UTI da MESM no ano de 2015. (n=70)

\begin{tabular}{|lcc|}
\hline Ensino superior completo & 4 & $5,7 \%$ \\
\hline Ensino médio completo & 20 & $28,5 \%$ \\
\hline $\begin{array}{l}\text { Ensino fundamental } \\
\text { completo }\end{array}$ & 38 & $54,2 \%$ \\
\hline Procedência & 30 & \\
\hline Maceió & 40 & $42,8 \%$ \\
\hline Interior & & $57,1 \%$ \\
\hline Paridade & 34 & \\
\hline Primigesta & 12 & $48,5 \%$ \\
\hline Secundigesta & 24 & $17,1 \%$ \\
\hline Multigesta & & $34,2 \%$ \\
\hline
\end{tabular}

Ao analisar os principais diagnósticos da admissão na UTI Materna, verificou-se um predomínio das sindromes hipertensivas da gravidez 55,7\%, síndromes hemorrágicas $14,2 \%$, cardiopatia $11,4 \%$. Outros diagnósticos menos frequentes, correspondem à infecção puerperal $7,1 \%$, crise asmática $5,7 \%$, e outras causas $5,7 \%$ (Tabela 2 ).
Tabela 2. Principais diagnósticos da admissão na UTI da MESM no ano de 2015. (n=70)

\begin{tabular}{|lcc|}
\hline \multicolumn{1}{|c}{$\begin{array}{c}\text { Diagnóstico da } \\
\text { admissão }\end{array}$} & N & $\%$ \\
\hline Sindromes hipertensivas & 39 & $55,7 \%$ \\
\hline Sindromes hemorrágicas & 10 & $14,2 \%$ \\
\hline Cardiopatia & 8 & $11,4 \%$ \\
\hline Infecção puerperal & 5 & $7,1 \%$ \\
\hline Crise asmática & 4 & $5,7 \%$ \\
\hline Outras causas & 1 & 2 \\
\hline
\end{tabular}

Referente às formas clínicas da síndrome hipertensiva, observou-se $33,3 \%$ casos de hipertensão gestacional, 15,3\% de pré-eclâmpsia, 35,8\% de eclâmpsia e 15,3\% de síndrome Hellp (Tabela 3). Com relação à classificação do quadro hemorrágico, $20 \%$ dos casos de descolamento prematuro de placenta, 30\% atonia uterina, 30\% abortamento e $20 \%$ gravidez ectópica (Tabela 4).

Tabela 3. Formas clinicas da sindrome hipertensiva na UTI da MESM no ano de 2015. (n=39)

\begin{tabular}{|lcc|}
\hline $\begin{array}{l}\text { Formas clinicas da } \\
\text { sindrome hipertensiva }\end{array}$ & N & $\%$ \\
\hline Hipertensão gestacional & 13 & $33,3 \%$ \\
\hline Pré-eclâmpsia & 6 & $15,3 \%$ \\
\hline Eclâmpsia & 14 & $35,8 \%$ \\
\hline Sindrome Hellp & 6 & $15,3 \%$ \\
\hline
\end{tabular}

Tabela 4. Sindromes hemorrágicas na UTI da MESM no ano de 2015. $(n=10)$

\begin{tabular}{|lcc|}
\hline Quadro hemorrágico & N & $\%$ \\
\hline $\begin{array}{l}\text { Descolamento prematuro } \\
\text { de placenta }\end{array}$ & 2 & $20 \%$ \\
\hline Atonia uterina & 3 & $30 \%$ \\
\hline Abortamento & 3 & $30 \%$ \\
\hline Gravidez ectópica & 2 & $20 \%$ \\
\hline
\end{tabular}

Das 70 pacientes estudadas, 37\% foram admitidas durante a gestação, $56 \%$ no pós-parto e $7 \%$ após abortamento ou gravidez ectópica (Tabela 5). Das 39 pacientes admitidas no período de gestação, 46,1\% evoluíram para o parto durante o internamento. $O$ tipo de parto realizado entre as pacientes que foram admitidas no pós-parto, e as que evoluíram durante o internamento, revelou que $94,7 \%$ foi cesárea, enquanto 
5,2\% foi parto normal. Sendo o parto prematuro (abaixo de 37 semanas) mais frequente, $54,3 \%$ dos casos. (Tabela 6 ).

Tabela 5. Fase do ciclo gravídico-puerperal da admissão na UTI da MESM no ano de 2015. $(n=70)$

\begin{tabular}{lcc|}
\multicolumn{1}{|c}{ Fase } & N & $\%$ \\
\hline Gestação & 26 & 37 \\
\hline Pós-parto & 39 & 56 \\
\hline $\begin{array}{l}\text { Abortamento/gravidez } \\
\text { ectópica }\end{array}$ & 5 & 7 \\
\hline
\end{tabular}

Tabela 6. Dados obstétricos das pacientes internas na UTI da MSM no ano de 2015. ( $n=57)$

\begin{tabular}{|c|c|c|}
\hline Dados obstétricos & $\mathbf{N}$ & $\%$ \\
\hline \multicolumn{3}{|l|}{ Tipo de parto } \\
\hline Cesárea & 54 & $94,7 \%$ \\
\hline Normal & 3 & $5,2 \%$ \\
\hline \multicolumn{3}{|l|}{ Idade gestacional do parto } \\
\hline$<32 \mathrm{~s}$ & 17 & $29,8 \%$ \\
\hline $32-37 \mathrm{~s}$ & 14 & $24,5 \%$ \\
\hline$>37 \mathrm{~s}$ & 26 & $45,6 \%$ \\
\hline
\end{tabular}

O tempo de internamento das pacientes na Unidade de Terapia Intensiva foi no intervalo de 1-31 dias, com média de 4 dias, $87,1 \%$ com duração entre 1-4 dias, 7,1\% entre 5-10 dias e 5,7\% acima de 10 dias (Tabela 7).

Tabela 7. Duração do internamento na UTI da MESM no ano de 2015. $(n=70)$

\begin{tabular}{|c|c|c|}
\hline $\begin{array}{c}\text { Duração do } \\
\text { internamento }\end{array}$ & $\mathrm{N}$ & $\%$ \\
\hline 1-4 dias & 61 & $87,1 \%$ \\
\hline $5-10$ dias & 6 & $7,1 \%$ \\
\hline$>10$ dias & 4 & $5,7 \%$ \\
\hline
\end{tabular}

No que diz respeito a situação de alta $87,1 \%$ receberam alta para Enfermaria, em estado clínico melhorado, 5,7\% alta para o pré-parto, 5,7\% foram à óbito e 1,4\% para UTI geral (Tabela 8).
Tabela 8. Situação de alta da UTI da Maternidade Escola Santa Mônica no ano de 2015. (n=70)

\begin{tabular}{|lcc|}
\hline \multicolumn{1}{|l}{ Situação de alta } & $\mathbf{n}$ & $\%$ \\
\hline Enfermaria & 61 & $87,1 \%$ \\
\hline Pré-parto & 4 & $5,7 \%$ \\
\hline Óbito & 4 & $5,7 \%$ \\
\hline UTI Geral & 1 & $1,4 \%$ \\
\hline
\end{tabular}

\section{DISCUSSÃO}

O estudo mostrou que as pacientes admitidas na UTI materna se caracterizam na maioria por pacientes adultasjovens, sendo a média de idade 27 anos, 60\% se encontrava na faixa etária entre 20 e 34 anos de idade, resultado semelhante ao encontrado em outros estudos, o que muito provavelmente decorre do fato de que a clientela da UTI obstétrica é constituída por mulheres na fase reprodutiva $(3,8)$. Esse resultado pode ser relacionado com a paridade das pacientes, onde o estudo revelou 48,5\% de primigesta, enfatizando assim a relação entre idade, fase reprodutiva e paridade. Esse resultado é contraditório se comparado ao estudo de Amorim et $\mathrm{al}^{3}$ onde revelou que a maioria das pacientes internas na UTI materna eram multigesta (38\%).

Quanto a procedência a maioria $57,1 \%$ das pacientes do estudo é de cidades do interior do estado, resultado já esperado pelo fato da Maternidade onde foi realizado a pesquisa ser uma unidade de atendimento público de referência para todo o estado. Fato que se corrobora no estudo de Medeiros et $\mathrm{al}^{8}$ onde $47,5 \%$ das pacientes eram procedentes do interior do Piauí.

No presente estudo as principais causas de internamento na UTI da MESM foram sindromes hipertensivas da gravidez $55,7 \%$, síndromes hemorrágicas $14,2 \%$, cardiopatia $11,4 \%$, dados que diferem um pouco da maioria dos estudos já realizados, que revelam as principais causas de internamento em unidades de terapia intensiva sendo pré-eclâmpsia, hemorragia e sepse ${ }^{3,8}$. O fato das cardiopatias estarem em terceiro lugar ao invés da sepse, como a maioria dos estudos, se explica pela rotina da instituição onde essas pacientes passam as primeiras horas do pós-parto na UTI materna como medida profilática.

A fase do ciclo gravídico puerperal no internamento com maior frequência foi no pós-parto 56\%, o que vai de acordo com a literatura, que demonstra os internamentos na terapia intensiva no puerpério. $\bigcirc$ que corrobora com os estudos de Amorim et al e Medeiros et $\mathrm{al}^{3,8}$ que traz os percentuais $87 \%$ e $69,8 \%$ de internamentos no pós-parto. Como justificativa, são sugeridas, a perda sanguínea que ocorre no parto, levando a descompensação hemodinâmica, e o adiamento da transferência das pacientes para UTI'3,8 
A taxa de cesariana na pesquisa chegou aos $97,4 \%$ dos casos, no estudo de Amorim3 a taxa foi $65 \%$, já no estudo de Medeiros et al8 85,6\%. A taxa elevada de cesárea é justificada em parte pela gravidade das complicações, impondo, em geral, o término da gestação em fase precoce, com condições cervicais desfavoráveis e/ou comprometimento do bemestar fetal, isso indica a escolha pela via de parto levando em consideração a gravidade do quadro. Taxas de cesariana iguais ou superiores a 70\% são descritas na literatura, e na Ásia já foram relatadas taxas de até $95 \%{ }^{3,8}$. Porém segundo a OMS a indicação de cesárea em estabelecimentos hospitalares para atenção a Gestante de Alto Risco é torno de 25\% para as Américas, devendo trabalhar para a taxa de cesárea de $30 \%$ no geral ${ }^{\text {. }}$.

O tempo de internamento das pacientes que tiveram alta, foi no intervalo entre um e 31 dias, com média em torno de quatro dias. Essa duração de internamento foi aproximada à relatada pelo estudo de Amorim et $\mathrm{al}^{3}$ que mostrou uma média de cinco dias. Essa duração reflete, possivelmente, o tempo necessário para recuperação pós-parto das doenças obstétricas como pré-eclâmpsia/eclâmpsia, sindrome HELLP ${ }^{3}$

A taxa de óbito encontrada foi 5,7\% um pouco elevada se comparada à estudos brasileiros e canadenses que tem uma taxa de mortalidade materna em aproximadamente $2,4 \% 3$. Segundo dados do DATASUS no ano de 2015 a taxa de mortalidade materna no Brasil foi de $2,16 \%{ }^{10}$.

O estudo apresentou algumas limitações, entre elas merece destaque a ausência de anotações nos prontuários.

\section{CONCLUSÃO}

Neste estudo verificou-se que as sindromes hipertensivas associada à gestação foi a maior causa de internamento na UTI materna. Referente às características dessas mulheres, a maioria era jovem, com média de 27 anos, primigestas, procedentes do interior de Alagoas, e com baixo grau de escolaridade. $O$ parto prematuro (abaixo de 37 semanas) foi frequente $54,3 \%$ dos casos e, independentemente da idade gestacional, o tipo de parto mais realizado foi a cesariana, em $94,7 \%$ das pacientes.

A duração de internamento foi 1-31 dias, com média de 4 dias. No que diz respeito a situação de alta a frequência global que receberam alta para a enfermaria com condição cínica estável foi alta. Sendo a taxa de óbito materno 5,7\%.

Conclui-se o quando a assistência obstétrica no estado de Alagoas precisa avançar para diminuir o número de óbitos maternos, e reduzir a taxa de cesárea mesmo em uma instituição referência para gestação de alto risco. É preciso profissionais e serviços qualificado para identificar precocemente alterações no ciclo gravídico puerperal, para assim evitar complicações que levem ao internamento na UTI, e consequências drásticas de morbi-mortalidade materna.

É válido ressaltar a relevância da assistência pré-natal de qualidade, de fácil acesso, pois é de suma importância para o reconhecimento precoce dos fatores de risco que levam a morbidade e mortalidade materna, permitindo, dessa forma, que intervenções apropriadas sejam implementadas. Ao priorizar a atenção básica, as causas previsiveis e evitáveis serão reduzidas e com isso a diminuição das complicações e o número de internações na UTI materna. É notório também a relevância da Unidade de Terapia Intensiva específica para a mulher no ciclo gravídico puerperal. Isso ajuda na melhoria da qualidade da assistência e principalmente a redução da morbimortalidade dessas pacientes. 


\section{REFERÊNCIAS}

1. Santos AG, Neto AMC, Nery IS. Assistência de enfermagem a mulheres com necessidades de cuidados intensivos durante o ciclo gravidico-puerperal. Revista Rede de Cuidados em Saúde [Internet]. 2016 [Cited 2017 Apr 30]. Available from: http://publicacoes.unigranrio.com.br/index.php/rcs/article/view/2707.

2. Amorim MMR, Katz L, Ávila MB, Araújo DE, Valença M, Albuquerque CJM, et al. Perfil das admissões em uma unidade de terapia intensiva obstétrica de uma maternidade brasileira. Rev Bras Saúde Matern Infant [Internet] 2006.6(Supl. 1): s55-s62. [Cited 2017 Apr 16]. Available from: http://www.scielo.br/scielo. php?script=sci_arttext\&pid=S1519-.

3. Coelho MAL, Katz L, Coutinho I, Hofmann A, Miranda L, Amorim M. Perfil de mulheres admitidas em uma UTI obstétrica por causas não obstétricas. Rev Assoc Med Bras. [Internet] 2012 [Cited 2017 Jan 20]:58(2):160-7. Available from: http://www.sciencedirect.com/science/article/pii/SO104423012704906.

4. Organização Mundial da Saúde. Classificação estatística internacional de doenças e problemas relacionados à saúde. Décima Revisão (CID-10). 8.a ed. São Paulo: Edusp; 2000.

5. Carvalho ML, Almeida CAL, Marques AKL, et al. Prevenção da mortalidade materna no pré-natal: uma revisão integrativa. R. Interd. v. 8, n. 2, p. 178-184, abr. mai. jun. 2015 [Internet] 2015. [Cited 2017 Jan 27] Available from: http://revistainterdisciplinar. uninovafapi.edu.br/index.php/revinter/article/view/733.

6. Ruiz MT, Wysocki AD. Editorial: Meta do milênio para a mortalidade materna: onde vamos chegar? Enfermagem Brasil [Internet]. 2016 [Cited 2017 Jan 09]. 15(3):114-5. Available from: http:// www.portalatlanticaeditora.com.br/index.php/enfermagembrasil/article/view/175/1543.
7. Dias JMG, Oliveira APS, Cipolotti R, Monteiro BKSM, Pereira RO. Mortalidade materna. Rev Médica de Minas Gerais [Internet]. 2015 [Cited 2017 Feb 10]. V 25.2. Available from: http://www. rmmg.org/artigo/detalhes/1771.

8. Medeiros TMC, Araújo KRS, Passos AC, et al. Perfil clínico epidemiológico das pacientes admitidas na unidade de terapia intensiva obstétrica de uma maternidade pública. Revista Eletrônica Gestão \& Saúde [Internet]. 2015 [Cited 2017 Jan 24] Vol.06, №. 02, p. 1601- 12. Available from: https://periodicos.ufpe.br/ revistas/revistaenfermagem/article/view/11455.

9. BRASIL, NOTA TÉCNICA 31 2012- CONASS [Internet]. 2012 [Cited 2017 Apr 02]. Available from: http://www.conass.org.br/ biblioteca/wp-content/uploads/2012/01/NT-31_2012-Atenc\%CC\%A7a\%CC\%83o-a\%CC\%80-Saude-Gestac\%CC\%A7a\%CC\%83o-de-Alto-Risco-final.pdf

10. Brasil. Ministério da Saúde. Departamento de informática do sistema único de saúde - DATASUS [Internet]. [Cited 2017 Mar 20]. Available from: http://tabnet.datasus.gov.br/cgi/tabcgi. exe?sim/cnv/pmatlouf.def. 